\title{
DS-CDMA Cellular Systems Performance with Base Station Assignment, Power Control Error, and Beamforming over Multipath Fading
}

\author{
Mohamad Dosaranian Moghadam ${ }^{1}$, Hamidreza Bakhshi², and Gholamreza \\ Dadashzadeh $^{2}$ \\ ${ }^{1}$ Department of Electrical Engineering, Islamic Azad University, Qazvin Branch, \\ Qazvin, Iran \\ m_dmoghadameqiau.ac.ir \\ ${ }^{2}$ Department of Electrical Engineering, Shahed University, Tehran, Iran \\ bakhshi@shahed.ac.ir \\ gdadashzadeheshahed.ac.ir
}

\begin{abstract}
The interference reduction capability of antenna arrays, base station assignment and the power control algorithms have been considered separately as means to increase the capacity in wireless communication networks. In this paper, we propose base station assignment method based on minimizing the transmitter power (BSA-MTP) technique in a direct sequence-code division multiple access (DS-CDMA) receiver in the presence of frequency-selective Rayleigh fading and power control error (PCE). This receiver consists of constrained least mean squared (CLMS) algorithm, matched filter (MF), and maximal ratio combining $(M R C)$ in three stages. Also, we present switched-beam (SB) technique in the first stage of the $R A K E$ receiver for enhancing signal to interference plus noise ratio (SINR) in DS-CDMA cellular systems. The simulation results indicate that BSA-MTP technique can significantly improve the network bit error rate (BER) in comparison with the conventional case. Finally, we discuss on three parameters of the PCE, number of resolvable paths, and channel propagation conditions (path-loss exponent and shadowing) and their effects on capacity of the system via some computer simulations.
\end{abstract}

\section{KEYWORDS}

Adaptive beamforming, base station assignment, DS-CDMA, power control error, matched filter, maximal ratio combining

\section{INTRODUCTION}

Systems utilizing code-division multiple access (CDMA) are currently being deployed around the country and around the world in response to the ever increasing demand for cellular/personal communications services. Extensive research has been published on the performance analysis of CDMA systems. Fading is among the major factors affecting the performance of such systems. Fading is generally characterized according to its effect over a geographical area. Large-scale fading consists of path loss and shadowing, the latter term referring to fluctuations in the received signal mean power. Large-scale fading is affected by prominent terrain contours between the transmitter and receiver. Small-scale fading is the common reference to the rapid changes in signal amplitude and phase over a small spatial separation. In this work, the combined effect of large- and small-scale fading are considered. The small-scale fading is assumed to be governed by the Rayleigh distribution (Rayleigh fading) [1], [2].

Besides fading, CDMA systems are susceptible to the near-far problem. It is well known that in order to fully exploit the potential advantage of CDMA systems, power control is required to counteract the effects of the near-far problem. The CDMA system capacity is maximized if each 
mobile transmitter power level is controlled so that its signal arrives at the base station (BS) with the minimum required signal to interference plus noise ratio (SINR) [2]-[7]. However, when applying power control in practice the performance is restricted by a number of limitations and therefore, perfect power control cannot be achieved. The issue of the effect of power control errors on CDMA systems has received a great deal of attention over the last few years [2]-[4]. Accordingly, in this paper, we consider the effect of power control error (PCE) on direct-sequence (DS)-CDMA cellular systems.

Also diversity is one effective technique for enhancing the SINR for wireless networks. Diversity exploits the random nature of radio propagation by finding independent (or, at least, highly uncorrelated) signal paths for communication. If one radio path undergoes a deep fade, another independent path may have a strong signal. By having more than one path to select from, the SINR at the receiver can be improved. The diversity scheme can be divided into three methods: 1) the space diversity; 2) the time diversity; 3) the frequency diversity. In these schemes, the same information is first received (or transmitted) at different locations (or time slots/frequency bands). After that, these signals are combined to increase the received SINR. The antenna array is an example of the space diversity, which uses a beamformer to increase the SINR for a particular direction [8]-[10]. In this work, we use constrained least mean squared (CLMS) algorithm and switched-beam (SB) technique for the space diversity.

To improve the performance of cellular systems, base station assignment (BSA) technique can be used. In the base station assignment, a number of base stations are potential receivers of a mobile transmitter. Here, the objective is to determine the assignment of users to base stations which minimizes the allocated mobile powers [11]-[14]. In simple mode and in multiple-cell systems, the user is connected to the nearest base station. This way is not optimal in cellular systems under the shadowing and multipath fading channels and can increase the system BER. In this paper, we present base station assignment method based on minimizing the transmitter power (BSA-MTP) for decreasing the BER in all cells [15]-[17].

The goal of this paper is to extend the works in [15]-[19] by considering joint multiple-cell system, BSA-MTP technique, and PCE. In [15]-[17], we proposed the BSA-MTP technique in DS-CDMA systems in multipath fading channels without considering the PCE. Also in [18] and [19], a RAKE receiver in single-cell system was proposed in the presence of frequency-selective Rayleigh fading channel, and the conventional BSA was considered.

In this work, the performance analysis of DS-CDMA system in frequency-selective Rayleigh fading channel has been studied. If the delay spread in a multipath channel is larger than a fraction of a symbol, the delayed components will cause inter-symbol interference (ISI). Adaptive receiver beamforming schemes have been widely used to reduce both co-channel interference (CCI) and ISI and to decrease the bit error rate (BER) by adjusting the beam pattern such that the effective SINR at the output of the beamformer is optimally increased [20].

In this paper, a RAKE receiver in DS-CDMA system is analyzed in three stages according to Figure 1 [18]. In the first stage, this receiver uses CLMS adaptive beamforming algorithm to find optimum antenna weights assuming perfect estimation of the channel parameters (direction, delay, and power) for the desired user. The desired user resolvable paths' directions are fed to the beamformer to reduce the inter-path interference (IPI) from other directions. Also, the RAKE receiver uses conventional demodulation in the second stage and conventional maximal ratio combining (MRC) in the third stage to reduce multiple access interference (MAI) and the other interferences. Reducing the MAI and CCI will further decrease the system BER.

The organization of the remainder of this paper is as follows. The system model is given in Section 2. The RAKE receiver structure is described in Section 3. In Section 4, we propose the BSA-MTP technique. In Section 5, we present the SB technique. Finally, simulation results and conclusions are given in Section 6 and Section 7, respectively. 
International Journal of Computer Networks \& Communications (IJCNC) Vol.3, No.1, January 2011

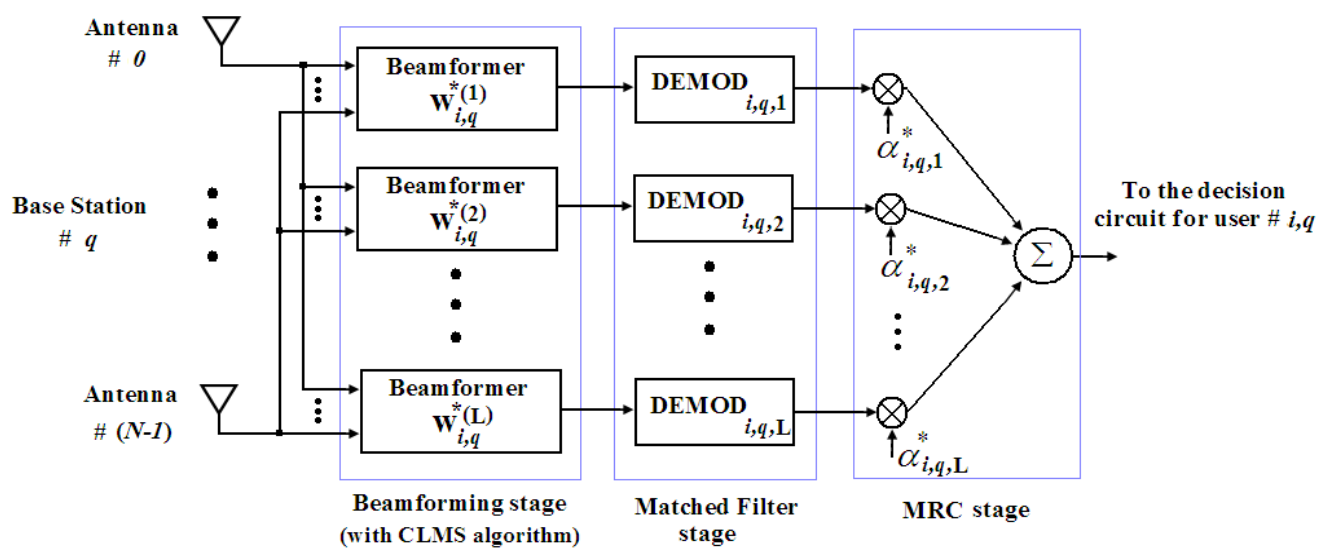

Figure 1. Block diagram of a three-stage RAKE receiver in DS-CDMA system [18]

\section{SYSTEM MODEL}

In this paper, we focus on the uplink communication paths in a DS-CDMA cellular system. The channel is modeled as a frequency selective channel with Rayleigh distribution and lognormal distributed shadowing. Initially, we consider $L$ paths for each link that optimally combined through a RAKE receiver according to Fig. 1. Also, we assume that there are $M$ active base stations in the network, with $K_{m}$ users connected to $m$ th base station. At each base station, an antenna array of $S$ sensors and $N$ weights is employed, where $S=N$, to receive signals from all users. Also, for simplicity we assume a synchronous DS-CDMA scheme and BPSK modulation in order to simplify the analysis of proposed technique. Additionally, in this paper we assume a slow fading channel (the channel random parameters do not change significantly during the bit interval). Hence, the received signal in the base station $q$ and sensor $s$ from all users can be written as [11], [18], [21]

$$
\begin{aligned}
r_{q, s}(t)= & \sum_{k} \sqrt{P \lambda_{k, m} \Gamma_{k}(x, y)} \sum_{l=1}^{L} \alpha_{k, m, l} b_{k, m}\left(t-\tau_{k, m, l}\right) c_{k, m}\left(t-\tau_{k, m, l}\right) \\
& \times \exp \left(-j 2 \pi s d \sin \theta_{k, m, l} / \lambda\right)+n(t)
\end{aligned}
$$

where $P=E_{b} / T_{b}$ represents the received signal power of all users within cell $q$ in the presence of perfect power control, where $E_{b}$ and $T_{b}$ are the energy per bit and bit period for all users, respectively. The variable $\lambda_{k, m}$ is PCE for user $k$ in cell $m$ (user $k, m$ ), which is assumed to follow a log-normal distribution and thus it can be written as $\lambda_{k, m}=10^{v_{k, m}} / 10$, where $v_{k, m}$ is a Gaussian random variable with mean 0 and variance $\sigma_{v}^{2}$ for all users [4]. Also, $c_{k, m}(t)$ is the pseudo noise (PN) chips of user $k, m$ with a chip period of $T_{c} ; b_{k, m}(t)$ is the information bit sequence of user $k, m$ with a bit period of $T_{b}=G T_{c}$ where $G$ is processing gain; $\tau_{k, m, l}$ is the $l$ th path time delay for user $k, m ; \theta_{k, m, l}$ is the direction of arrival (DoA) in the $l$ th path for user $k, m ; \alpha_{k, m, l}$ is the complex Gaussian fading channel coefficient from the $l$ th path of user $k, m$; $\lambda$ is signal wavelength; $d$ is the distance between the antenna elements that for avoid the spatial aliasing should be defined as $d=0.5 \lambda ; n(t)$ is an additive white Gaussian noise (AWGN) process with a two-sided power spectral density (PSD) of $N_{0} / 2$. Also for conventional BSA technique, $\Gamma_{k}(x, y)$ is defined as 
International Journal of Computer Networks \& Communications (IJCNC) Vol.3, No.1, January 2011

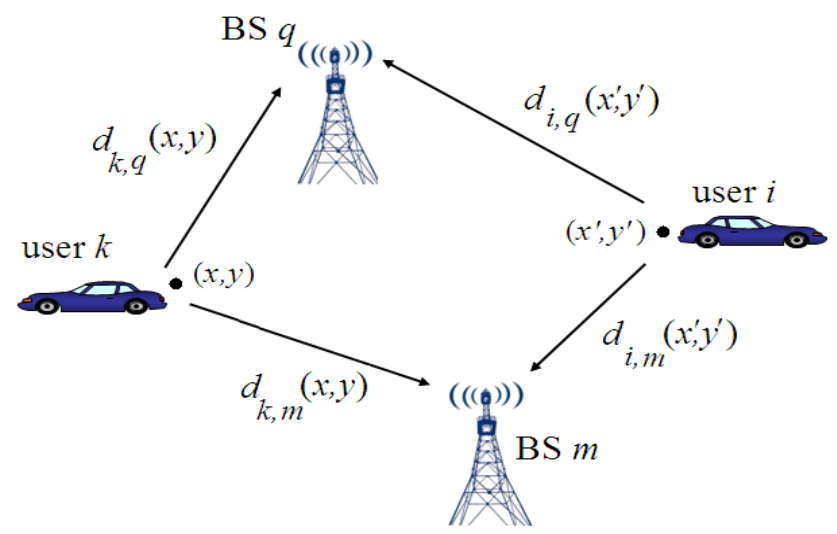

Figure 2. The distance between two pairs of mobile transmitters and base station receivers [11]

$$
\Gamma_{k}(x, y)=\left\{\begin{array}{cc}
1 & ; k \in S_{\mathrm{BS} q} \\
\frac{\min _{m \in \Theta_{k}}\left\{d_{k, m}^{L_{\alpha}}(x, y) 10^{\xi_{k, m} / 10}\right\}}{d_{k, q}^{L_{\alpha}}(x, y) 10^{\xi_{k, q} / 10}} ; k \in S_{o} &
\end{array}\right.
$$

where $L_{\alpha}$ is path-loss exponent; $d_{k, m}(x, y)$ and $d_{k, q}(x, y)$ are the distance between user $k$ and $\mathrm{BS} m$ and $\mathrm{BS} q$, respectively (see Figure 2); Also the variable $\Theta_{k}$ defined the set of the nearest BSs to user $k ; \xi_{k, m}$ is a random variable modelling the shadowing between user $k$ and $\mathrm{BS} m ; S_{\mathrm{BS} q}$ is the set of users that connected to $\mathrm{BS} q$ and $S_{o}$ is the set of users that not connected to $\mathrm{BS} q[4]$.

Also, it should be mentioned that the transmitted power of user $k$ in cell $m$ to BS $m$ in the case of the PPC is given by

$$
p_{k, m}=d_{k, m}^{L_{\alpha}}(x, y) 10^{\xi_{k, m} / 10} \times P
$$

Accordingly, the received signal in the base station $q$ in sensor $s$ for user $i, q$ is given by [18]

$$
r_{i, q, s}^{\prime}(t)=\sum_{l=1}^{L} \sqrt{P \lambda_{i, q}} b_{i, q}\left(t-\tau_{i, q, l}\right) c_{i, q}\left(t-\tau_{i, q, l}\right) \alpha_{i, q, l} \exp \left(-j 2 \pi s d \sin \theta_{i, q, l} / \lambda\right)+I_{i, q, s}(t)+n(t)
$$

where $I_{i, q, s}(t)$ is the interference for user $i, q$ in sensor $s$ and can be shown to be

$$
\begin{aligned}
I_{i, q, s}(t)= & \sum_{m=1}^{M} \sum_{\substack{k=1 \\
k, m \neq i, q}}^{K_{m}} \sum_{l=1}^{L} \sqrt{P \lambda_{k, m} \Gamma_{k}(x, y)} b_{k, m}\left(t-\tau_{k, m, l}\right) c_{k, m}\left(t-\tau_{k, m, l}\right) \alpha_{k, m, l} \\
& \times \exp \left(-j 2 \pi s d \sin \theta_{k, m, l} / \lambda\right)
\end{aligned}
$$

where $K_{m}$ is the number of users in cell $m$ and $M$ is the number of base stations/cells.

\section{Rake Receiver Performance Analysis}

The RAKE receiver structure in the DS-CDMA system is shown in Figure 1. The received signal is spatially processed by a beamforming circuit with CLMS algorithm, one for each resolvable path ( $L$ beamformers). The resultant signal is then passed on to a set of parallel 
matched filters (MFs), on a finger-by-finger basis. Also, the output signals from the $L$ matched filters are combined according to the conventional MRC principle and then are fed into the decision circuit of the desired user.

\subsection{Constrained LMS Algorithm}

It is well known that an array of $N$ weights has $N-1$ degree of freedom for adaptive beamforming [18], [21]. This means that with an array of $N$ weights, one can generates $N-1$ pattern nulls and a beam maximum in desired directions. From Eq. (5), it is clear that the number of users is $K_{u}=\sum_{m=1}^{M} K_{m}$ and the number of interference signals is $L K_{u}-1$. To null all of these interference signals; one would have to have $L K_{u}$ weights, which is not practical. So, we focus only on the $L$ paths of the desired user. Thus, the minimum number of the antenna array weights is $L$ where, typically, $L$ varies from 2 to 6 [18].

In this paper, we use the CLMS adaptive beamforming algorithm. This algorithm is a gradient based algorithm to minimize the total processor output power, based on the look direction constraint. The adaptive algorithm is designed to adapt efficiently in agreement with the environment and able to permanently preserve the desired frequency response in the look direction while minimizing the output power of the array. The combined form of the constraint is called constraint for narrowband beamforming [20], [22].

This form consider a narrowband beamformer where the narrowband signal from each element of smart antenna are multiplied by the complex weight calculated by using narrowband adaptive beamforming algorithm, and then summed to produce the output of the array. The definition of the complex weights of this beamformer in the $n$th iteration for user $i, q$ in the $j$ th path is as follows [21], [23].

$$
\mathbf{w}_{i, q}^{(j)}(n)=\left[w_{i, q, 0}^{(j)}(n) w_{i, q, 1}^{(j)}(n) \ldots w_{i, q, N-1}^{(j)}(n)\right]^{T}
$$

Accordingly, the output of the array in the $n$th iteration in the $j$ th path for user $i, q$ is given by

$$
y_{i, q}^{(j)}(n)=\mathbf{w}_{i, q}^{(j)}(n)^{H} \mathbf{r}_{i, q}^{\prime}(n)
$$

where $\mathbf{r}_{i, q}^{\prime}=\left[\begin{array}{llll}r_{i, q, 0}^{\prime} & r_{i, q, 1}^{\prime} & \ldots & r_{i, q, N-1}^{\prime}\end{array}\right]^{T}$.

The expected output power of the array in the $n$th iteration is given by

$$
\begin{aligned}
\mathrm{E}\left(\left|y_{i, q}^{(j)}(n)\right|^{2}\right) & =\mathrm{E}\left(y_{i, q}^{(j)}(n) y_{i, q}^{(j)}(n)^{*}\right)=\mathrm{E}\left(\mathbf{w}_{i, q}^{(j)}(n)^{H} \mathbf{r}_{i, q}^{\prime}(n) \mathbf{r}_{i, q}^{\prime}(n)^{H} \mathbf{w}_{i, q}^{(j)}(n)\right) \\
& =\mathbf{w}_{i, q}^{(j)}(n)^{H} \mathbf{R}_{r^{\prime} r^{\prime}} \mathbf{w}_{i, q}^{(j)}(n)
\end{aligned}
$$

where $\mathrm{E}($.$) is denoted the expectation and \mathbf{R}_{r^{\prime} r^{\prime}}$ is the correlation matrix of the received vector $\mathbf{r}_{i, q}^{\prime}(n)$.

A real-time CLMS algorithm for determining the optimal weight vector for user $i, q$ in the $j$ th path is [22], [23]:

$$
\left\{\begin{array}{l}
\mathbf{w}_{i, q}^{(j)}(n+1)=\mathbf{w}_{i, q}^{(j)}(n)+\mu g\left(\mathbf{w}_{i, q}^{(j)}(n)\right) \\
\mathbf{w}_{i, q}^{(j) H} \mathbf{a}_{i, q}^{(j)}\left(\theta_{i, q, j}\right)=1
\end{array}\right.
$$

where 
International Journal of Computer Networks \& Communications (IJCNC) Vol.3, No.1, January 2011

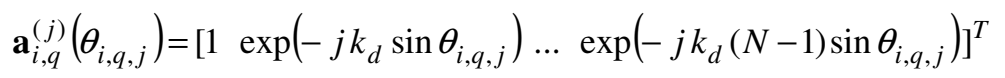

denotes spatial response of the array for user $i, q$ in the $j$ th path. Also in Eq. (9), $\mathbf{w}_{i, q}^{(j)}(n+1)$ is the new weight computed at the $(n+1)$ th iteration for user $i, q$ in the $j$ th path. Also, the variable scalar $\mu$ denotes a positive scalar (gradient step size) that controls the convergence characteristic of the algorithm, that is, how fast and how close the estimated weights approach the optimal weights, and $g\left(\mathbf{w}_{i, q}^{(j)}(n)\right)$ denotes an unbiased estimate of the gradient of the power surface $\left(\mathbf{w}_{i, q}^{(j)}(n)^{H} \mathbf{R}_{r^{\prime} r^{\prime}} \mathbf{w}_{i, q}^{(j)}(n)\right.$ which is the expected output power of the array) with respect to $\mathbf{w}_{i, q}^{(j)}(n)$ after the $n$th iteration. The algorithm is "constrained" because the weight vector satisfies the constraint at each iteration, that is $\mathbf{w}_{i, q}^{(j)}{ }^{H} \mathbf{a}_{i, q}^{(j)}\left(\theta_{i, q, j}\right)=1$. Rewrite the CLMS algorithm as follows [22].

$$
\mathbf{w}_{i, q}^{(j)}(n+1)=\boldsymbol{\beta}_{i, q}^{(j)}\left(\mathbf{w}_{i, q}^{(j)}(n)+\mu g\left(\mathbf{w}_{i, q}^{(j)}(n)\right)\right)+\frac{\mathbf{a}_{i, q}^{(j)}\left(\theta_{i, q, j}\right)}{N}
$$

where

$$
\boldsymbol{\beta}_{i, q}^{(j)}=\mathbf{I}-\frac{\mathbf{a}_{i, q}^{(j)}\left(\theta_{i, q, j}\right) \mathbf{a}_{i, q}^{(j)}\left(\theta_{i, q, j}\right)^{H}}{N}
$$

The gradient of $\mathbf{w}_{i, q}^{(j)}(n)^{H} \mathbf{R}_{r^{\prime} r^{\prime}} \mathbf{w}_{i, q}^{(j)}(n)$ with respect to $\mathbf{w}_{i, q}^{(j)}(n)$ is given by

$$
g\left(\mathbf{w}_{i, q}^{(j)}(n)\right) \stackrel{\Delta}{=}-\frac{\partial}{\partial \mathbf{w}_{i, q}^{(j) *}}\left(\mathbf{w}_{i, q}^{(j)}(n)^{H} \mathbf{R}_{r^{\prime} r^{\prime}} \mathbf{w}_{i, q}^{(j)}(n)\right)=-2 \mathbf{R}_{r^{\prime} r^{\prime}} \mathbf{w}_{i, q}^{(j)}(n)
$$

and its computation using this expression requires knowledge of $\mathbf{R}_{r^{\prime} r^{\prime}}$, which normally is not available in practice. For a standard LMS algorithm, an estimate of the gradient at each iteration is made by replacing $\mathbf{R}_{r^{\prime} r^{\prime}}$ by its noise sample $\mathbf{r}_{i, q}^{\prime}(n+1) \mathbf{r}_{i, q}^{\prime}(n+1)^{H}$ available at time instant $(n+1)$, leading to

$$
g\left(\mathbf{w}_{i, q}^{(j)}(n)\right)=-2 \mathbf{r}_{i, q}^{\prime}(n+1) y_{i, q}^{(j) *}(n)
$$

The CLMS is a fast convergence algorithm. However, it is drastically sensitive to the mismatch in the direction of arrival. Meanwhile, the weights estimated by the standard algorithm are sensitive to the signal power, requiring a lower step size in the presence of a strong signal for the algorithm to converge, which in turn regarding the decrease of mis-adjustment error, the convergence time is increased [22], [24].

It should be mentioned that for the antenna arrays weight vector in the CLMS algorithm and for $\operatorname{big} \mu$, will converge after a few iteration (is approximately equal to the number of beamformer weights, i.e., $n=N$ ) [24].

Accordingly, the output signal from the $j$ th beamformer $(j=1, \ldots, L)$ can be written as [18]

$$
y_{i, q}^{(j)}(t)=\sqrt{P \lambda_{i, q}} b_{i, q}\left(t-\tau_{i, q, j}\right) c_{i, q}\left(t-\tau_{i, q, j}\right) \alpha_{i, q, j}+\tilde{I}_{i, q}^{(j)}(t)+I_{i, q}^{(j)}(t)+n^{(j)}(t)
$$

where $n^{(j)}(t)$ is a zero mean Gaussian noise of variance $\sigma_{n}^{2}$ and $\tilde{I}_{i, q}^{(j)}(t)$, the IPI, is defined as 
International Journal of Computer Networks \& Communications (IJCNC) Vol.3, No.1, January 2011

$$
\tilde{I}_{i, q}^{(j)}(t)=\sum_{\substack{l=1 \\ l \neq j}}^{L} \sqrt{P \lambda_{i, q}} g_{i, q}^{(j)}\left(\theta_{i, q, l}\right) \alpha_{i, q, l} b_{i, q}\left(t-\tau_{i, q, l}\right) c_{i, q}\left(t-\tau_{i, q, l}\right)
$$

and $I_{i, q}^{(j)}(t)$, the MAI, is defined as

$$
I_{i, q}^{(j)}(t)=\sum_{m=1}^{M} \sum_{\substack{k=1 \\ k, m \neq i, q}}^{K_{m}} \sum_{l=1}^{L} \sqrt{P \lambda_{k, m} \Gamma_{k}(x, y)} g_{i, q}^{(j)}\left(\theta_{k, m, l}\right) \alpha_{k, m, l} b_{k, m}\left(t-\tau_{k, m, l}\right) c_{k, m}\left(t-\tau_{k, m, l}\right)
$$

where $g_{i, q}^{(j)}(\theta)$ is the magnitude response of the $j$ th beamformer for user $i, q$ toward the DoA $\theta$.

\subsection{Matched Filter Stage}

Using beamforming in the first stage, will reduce the IPI for the desired user and the MAI from the other users whose signals arrive at different angles from the desired user signal (out-beam interference). Now, in the second stage of the RAKE receiver, the output signal from the $j$ th beamformer is directly passes on to a filter matched to the desired user's signature sequence. The $j$ th matched filter output corresponding to the $n$th bit is [18]:

$$
z_{i, q}^{(j)}(n)=\sqrt{P \lambda_{i, q}} b_{i, q}(n) \alpha_{i, q, j}+\tilde{I}_{i, q}^{\prime(j)}(n)+I_{i, q}^{\prime(j)}(n)+n^{\prime(j)}(n)
$$

where

$$
\begin{gathered}
\tilde{I}_{i, q}^{\prime(j)}(n)=\frac{1}{T_{b}} \int_{(n-1) T_{b}+\tau_{i, q, j}}^{n T_{b}+\tau_{i, q, j}} \widetilde{I}_{i j,}^{(j)}(t) c_{i, q}\left(t-\tau_{i, q, j}\right) d t \\
I_{i, q}^{\prime(j)}(n)=\frac{1}{T_{b}} \int_{(n-1) T_{b}+\tau_{i, q, j}}^{n T_{b}+\tau_{i, q, j}} I_{i, q}^{(j)}(t) c_{i, q}\left(t-\tau_{i, q, j}\right) d t
\end{gathered}
$$

and

$$
n^{\prime(j)}(n)=\frac{1}{T_{b}} \int_{(n-1) T_{b}+\tau_{i, q, j}}^{n T_{b}+\tau_{i, q, j}} n^{(j)}(t) c_{i, q}\left(t-\tau_{i, q, j}\right) d t
$$

If we assume that the paths' delays from all users are less than the symbol duration $\left(\tau_{k, m, l}<T_{b}\right)$ for all users' signals on all paths, the $n$th bit IPI and MAI at the output of the $j$ th matched filter are expressed as

$$
\tilde{I}_{i, q}^{\prime(j)}(n)=\sum_{\substack{l=1 \\ l \neq j}}^{L} \sqrt{P \lambda_{i, q}} g_{i, q}^{(j)}\left(\theta_{i, q, l}\right) \alpha_{i, q, l} b_{i, q}(n) R_{i, i}\left(\tau_{i, q, j}-\tau_{i, q, l}\right)
$$

and 
International Journal of Computer Networks \& Communications (IJCNC) Vol.3, No.1, January 2011

$$
I_{i, q}^{\prime(j)}(n)=\sum_{m=1}^{M} \sum_{\substack{k=1 \\ k, m \neq i, q}}^{K_{m}} \sum_{l=1}^{L} \sqrt{P \lambda_{k, m} \Gamma_{k}(x, y)} g_{i, q}^{(j)}\left(\theta_{k, m, l}\right) \alpha_{k, m, l} b_{k, m}(n) R_{i, k}\left(\tau_{i, q, j}-\tau_{k, m, l}\right)
$$

where the autocorrelation function $R_{i, k}(\tau)$ is [1], [18]:

$$
R_{i, k}(\tau)=\frac{1}{T_{b}} \int_{T_{b}} c_{i, q}(t) c_{k, m}(t+\tau) d t
$$

If all users' delays are multiples of the chip period $\left(T_{c}\right)$, then

$$
R_{i, k}(\tau)=\frac{1}{G} \sum_{l_{1}=0}^{G-1} \sum_{l_{2}=0}^{G-1} c_{i, q}\left(l_{1}\right) c_{k, m}\left(l_{2}\right) R_{c}\left(\tau-\left(l_{1}-l_{2}\right) T_{c}\right)
$$

where the autocorrelation function $R_{c}(\tau)$ is:

$$
R_{c}(\tau)=\frac{1}{T_{b}} \int_{T_{b}} c(t) c(t+\tau) d t
$$

In the case of a maximal-length sequence (m-sequence) and for $0 \leq \tau \leq T_{b}$, we have [1]:

$$
R_{c}(\tau)= \begin{cases}1-\frac{|\tau|}{T_{c}}(1+1 / G) & ;|\tau| \leq T_{c} \\ -1 / G & ;|\tau| \geq T_{c}\end{cases}
$$

\subsection{Maximal Ratio Combining Stage}

Diversity combining has been considered as an efficient way to combat multipath fading because the combined SINR is increased compared with the SINR of each diversity branch. The optimum combiner is the MRC whose SINR is the sum of the SINR's of each individual diversity branch [1], [25].

After the finger-matched filter, the fingers' signals are combined according to the MRC principle in the third stage of the RAKE receiver. In this paper, we use the conventional MRC that the signal of user $i, q$ in the $j$ th path is combined using multiplying by the complex conjugate of $\alpha_{i, q, j}$.

The SINR in output of the RAKE receiver for user $i, q$ conditioned on $\alpha_{i, q, j}$ is given as [18], [25]

$$
\operatorname{SINR}_{i, q}(\alpha)=\sum_{j=1}^{L} \operatorname{SINR}_{i, q}^{(j)}(\alpha)
$$

where

$$
\operatorname{SINR}_{i, q}^{(j)}(\alpha)=\frac{P\left|\alpha_{i, q, j}\right|^{2} e^{\beta v_{i, q}}}{\mathrm{E}\left(\tilde{I}_{i, q}^{\prime(j)}\right)^{2}+\mathrm{E}\left(I_{i, q}^{\prime(j)}\right)^{2}+\mathrm{E}\left(n^{\prime(j)}\right)^{2}}
$$

is the SINR in output of the RAKE receiver in path $j$ for user $i, q$ and $\beta=\ln (10) / 10$. 
On the other hand, $\mathrm{E}\left[\lambda_{k, m}\right]$ for all users can be written as [26]

$$
\mathrm{E}\left[\lambda_{k, m}\right]=e^{\beta^{2} \sigma_{v}^{2} / 2}
$$

Now, using Eqs. (19)-(21), and (30), we can be rewritten the SINR in Eq. (29) as follows [27], [28].

$$
\operatorname{SINR}_{i, q}^{(j)}(\alpha)=\frac{\left|\alpha_{i, q, j}\right|^{2} e^{\beta v_{i, q}}}{\eta e^{\beta^{2} \sigma_{v}^{2} / 2}+\frac{0.5}{E_{b} / N_{0}}}
$$

where

$$
\begin{aligned}
\eta=\sum_{m=1}^{M} & \sum_{\substack{k=1 \\
k, m \neq i, q}}^{K_{m}} \bar{\Gamma}_{k}(x, y) \bar{\alpha}_{k, m, j}^{2} \sum_{l=1}^{L}\left|g_{i, q}^{(j)}\left(\theta_{k, m, l}\right)\right|^{2} R_{i, k}^{2}\left(\tau_{i, q, j}-\tau_{k, m, l}\right) \\
& +\bar{\alpha}_{i, q, j}^{2} \sum_{\substack{l=1 \\
l \neq j}}^{L}\left|g_{i, q}^{(j)}\left(\theta_{i, q, l}\right)\right|^{2} R_{i, i}^{2}\left(\tau_{i, q, j}-\tau_{i, q, l}\right)
\end{aligned}
$$

Also in Eq. (32), $\bar{\Gamma}_{k}(x, y)=\mathrm{E}\left(\Gamma_{k}(x, y)\right)$ and $\bar{\alpha}_{k, m, j}^{2}=\mathrm{E}\left(\left|\alpha_{k, m, j}\right|^{2}\right)$.

In order to perform the BER, we assume Gaussian approximation for the probability density function of interference plus noise. The conditional BER for a BPSK modulation is [1], [18]:

$$
\operatorname{BER}_{i, q}(\alpha)=Q\left(\sqrt{2 \times \operatorname{SINR}_{i, q}(\alpha)}\right)
$$

where

$$
Q(x)=\frac{1}{\sqrt{2 \pi}} \int_{x}^{\infty} \exp \left(-u^{2} / 2\right) d u
$$

\section{THE BSA-MTP TECHNIQUE}

The system capacity might be improved, if the users are allowed to switch to alternative base stations, especially when there are congested areas in the network. Obviously, when uplink performance is of concern, the switching should happen based on the total interferences seen by the base stations [14].

So far, we have considered the power control problem for a number of transmitter-receiver pairs with fixed assignments, which can be used in uplink or downlink in mobile communication systems. In an uplink scenario where base stations are equipped with antenna arrays, the problem of joint power control and beamforming, as well as base station assignment, naturally arises [11].

In this paper, we modify the BSA-MTP technique in multipath fading channel to support base station assignment as well. The modified technique can be summarized as follows [15]-[17].

1) Initially by the conventional BSA technique, each mobile connects to its base station, according to Eq. (2).

2) Estimate the weight vector for all users with the CLMS algorithm using Eq. (11). 


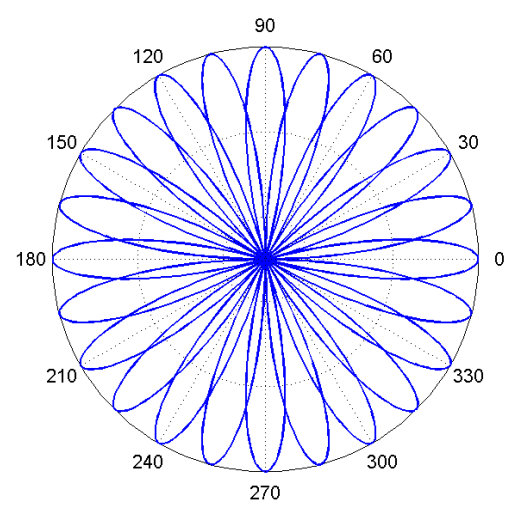

Figure 3. 36 beams in each base station with the SB technique

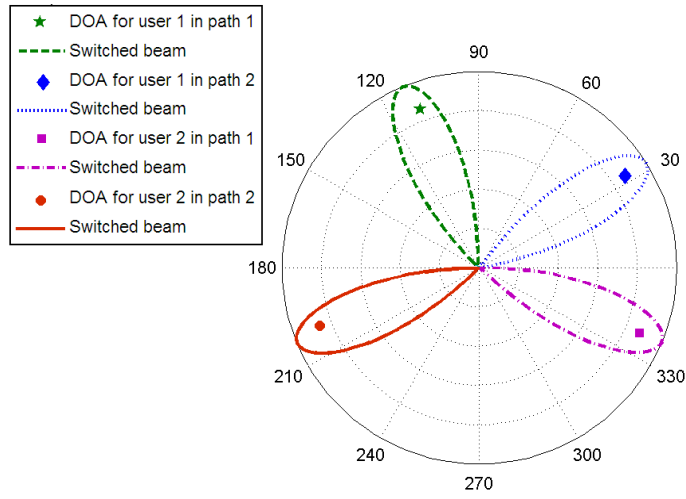

Figure 4. Select of beam for two users in two paths with the SB technique

3) Calculate the transmitted power of all users using Eq. (3).

4) Finally, $K_{r}=\left\lfloor K_{u} / M\right\rfloor$ users that their transmitted power is higher than the other users to be transferred to other base stations according to the following equation, where the function $\lfloor x\rfloor$ returns the integer portion of a number $x$.

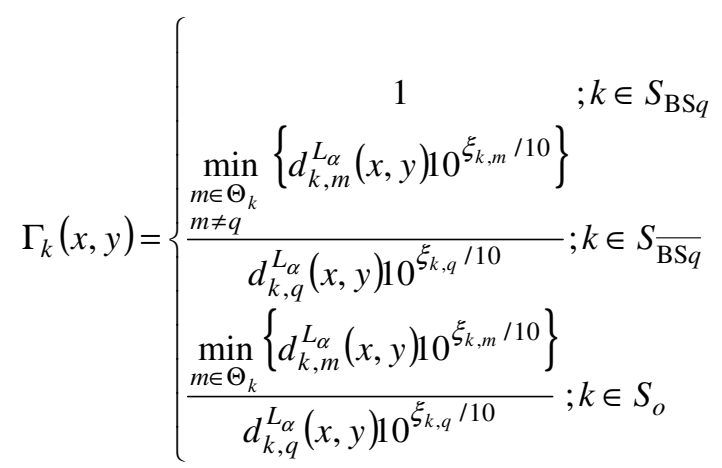

where $S_{\overline{\mathrm{BS} q}}$ is the set of users that are in cell $q$ but not connected to $\mathrm{BS} q$ [4].

It should be mentioned that the technique for users that are present in the border of cells, the BER can be effectively reduced.

\section{THE SWITCHED-BEAM TECHNIQUE}

One simple alternative to the fully adaptive antenna is the switched-beam architecture in which the best beam is chosen from a number of fixed steered beams. Switched-beam systems are technologically the simplest and can be implemented by using a number of fixed, independent, or directional antennas [29].

We list the conditions of the SB technique for this paper as follows [30].

1) According to Figure 3, beams coverage angle is $30^{\circ}$ and overlap between consecutive beams is $20^{\circ}$. Thus each base station has 36 beams.

2) According to Figure 4, each user can be use one beam for its each path to communicate with a base station at any time. 
International Journal of Computer Networks \& Communications (IJCNC) Vol.3, No.1, January 2011

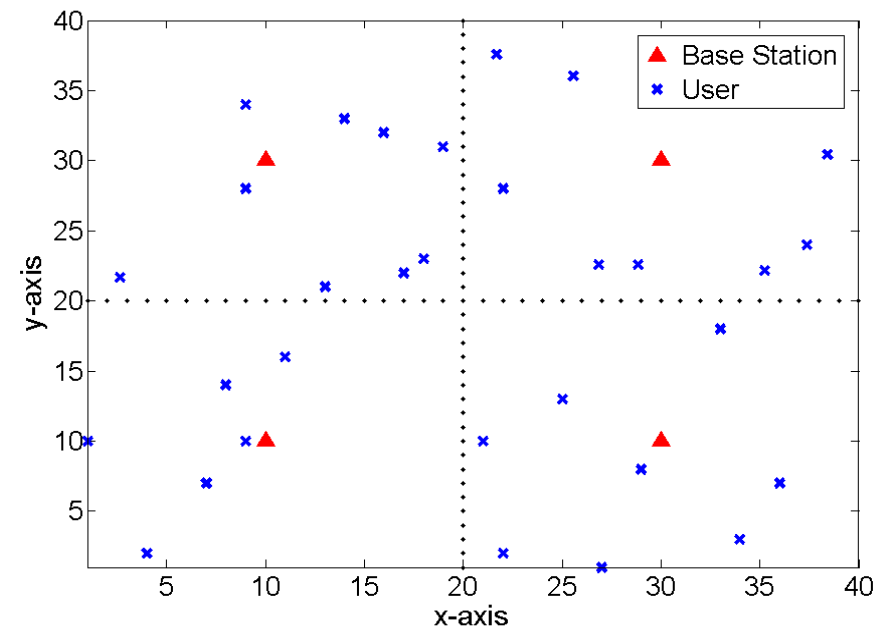

Figure 5. Location plot of base stations and users in four cells

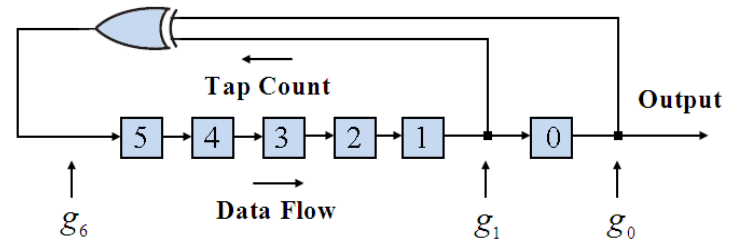

(a)

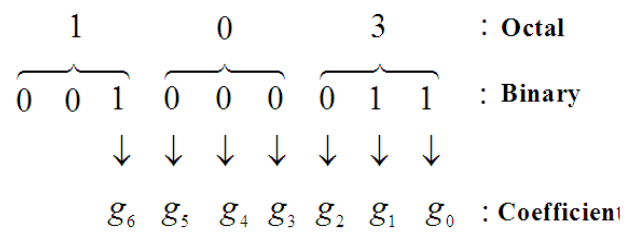

(b)

Figure 6. (a) Fibonacci feedback generator for LFSR polynomial $g(D)=1+D+D^{6}$ for sixstage shift register (b) Expanding the octal entry 103 into binary form [1]

\section{Simulation Results}

We consider $M=4$ base stations for a four-cell CDMA system on a $2 \times 2$ grid and size $(40 \times 40)$ as Figure 5 . We assume a uniform linear array of $S$ omni-directional antennas in each base station with antenna spacing $d=\lambda / 2$. Also, we assume the input data rate $T_{b}=9.6 \mathrm{Kbps}$; the number of antenna weights $N=3$; frequency-selective fading channel with $L=2$ resolvable propagation paths; variance of the complex Gaussian fading channel coefficient $\sigma_{\alpha}^{2}=4 \mathrm{~dB}$; path-loss component $L_{\alpha}=4$; variance of the log-normal shadow fading $\sigma_{\xi}^{2}=8 \mathrm{~dB}$; resolution $R=1$; initial value for weight vectors in the CLMS algorithm $\mathbf{w}(0)=\mathbf{0}$.

It also is assumed that the distribution of users in all cells is uniform.

In this paper, we use m-sequence generator with processing gain $G=64$ based on linear feedback shift register (LFSR) circuit using the Fibonacci feedback approach [1]. This structure is shown in Figure 6 (a). Also, according to [1], we use the sequence generated by the polynomial corresponding to the entry the octal representation of generator polynomial, ORGP= [103]* for a six-stage shift register. Figure 6 (b) shows expanding the octal entry 103 into binary form. Then, the LFSR polynomial is $g(D)=1+D+D^{6}$.

Figure 7 shows the average BER versus the signal to noise ratio (SNR) for different receivers (one, two, and three-stage receivers), $K_{u}=32$ active users, and a log-normally distributed PCE with $\sigma_{v}^{2}=4 \mathrm{~dB}$. It should be mentioned that in this simulation, $K_{r}=8$ users can be transferred to 
other base stations with the BSA-MTP technique. It is clear that, in MF only receiver (one-stage receiver) and in the case of the conventional BSA technique, we still have the error floor at high SNR. Using CLMS and MRC receiver (two-stage RAKE receiver) or CLMS, MF, and MRC receiver (the three-stage RAKE receiver as Figure 1) has a better performance than using MF only. Also, we observe that using the BSA-MTP technique in CLMS, MF, and MRC receiver, the average BER is lower than the conventional BSA technique. For example, at a SNR of $10 \mathrm{~dB}$, the average BER is 0.0194 for the three-stage RAKE receiver with the conventional BSA

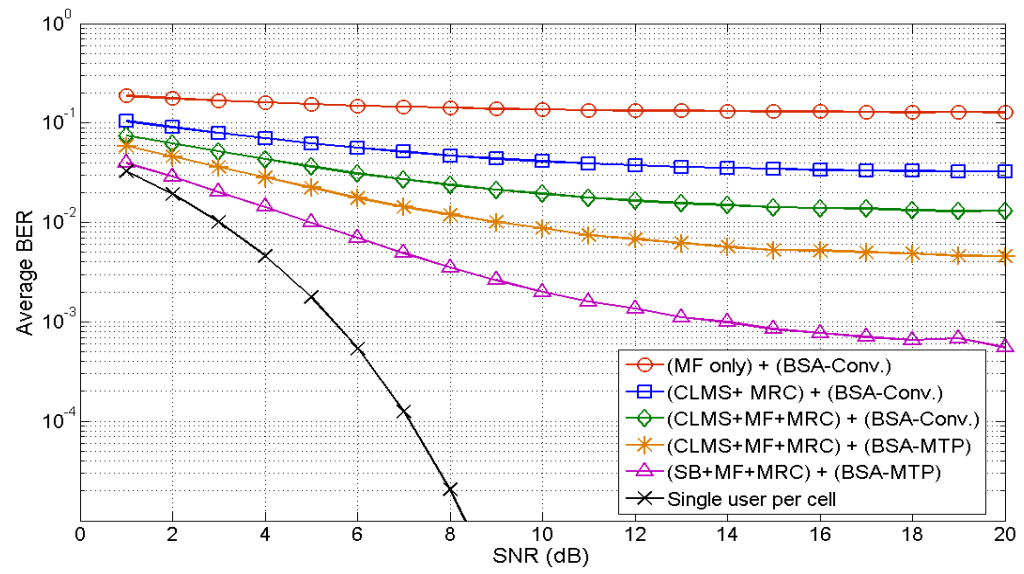

Figure 7. Average BER of all users versus the SNR for $\sigma_{v}^{2}=4 \mathrm{~dB}$ and $K_{u}=32$

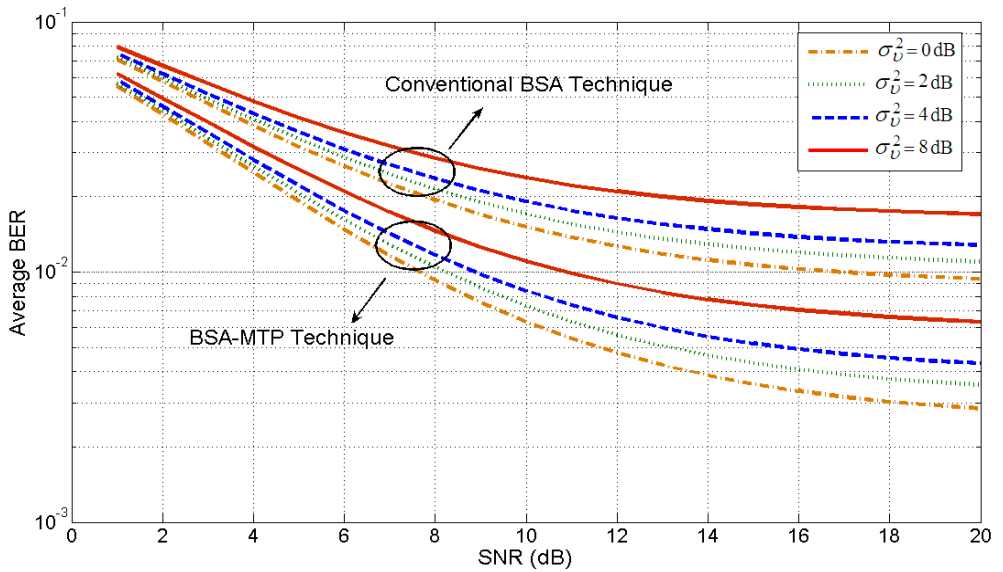

Figure 8. Average BER of all users versus the SNR for different values of $\sigma_{\text {. }}^{2}$

technique, while for the BSA-MTP technique, the average BER is 0.0087. Also it can be seen that the average BER in the CLMS algorithm is higher than the SB technique. In addition, we observe that using the BSA-MTP technique in SB, MF, and MRC receiver, the average BER is lower than other cases. For example, at a SNR of $12 \mathrm{~dB}$, the average BER using the BSA-MTP technique is 0.0014 for SB technique, while the average BER for CLMS algorithm is 0.0068. Also, it is clear that the MAI is not removed totally and the performance is still worse than the single user per cell bound. Results for the average BER versus the SNR for CLMS, MF, and MRC receiver and $K_{u}=32$ active users, and different values of $\sigma_{v}^{2}$ are provided in Figure 8. In this figure, similar to Figure 7, we observe that the average BER for the BSA-MTP technique is 
lower than the conventional BSA technique. Also it can be seen that the average BER for $\sigma_{v}^{2}=0 \mathrm{~dB}$ (perfect power control) is lower than the other cases [15]. For example, at a SNR of $10 \mathrm{~dB}$ and for the BSA-MTP technique, the average BER is 0.0063 for $\sigma_{v}^{2}=0 \mathrm{~dB}$, while for $\sigma_{v}^{2}=2 \mathrm{~dB}$ and $\sigma_{v}^{2}=8 \mathrm{~dB}$ the average BER are 0.0073 and 0.011 , respectively.

Figure 9 shows the average BER versus the number of active users $\left(K_{u}\right)$ for different receivers as Figure 7 and for SNR $=10 \mathrm{~dB}$, and $\sigma_{v}^{2}=4 \mathrm{~dB}$. At a BER of 0.01, CLMS, MF, and MRC receiver with the BSA-MTP technique support $K_{u}=36$ users, while for the conventional BSA

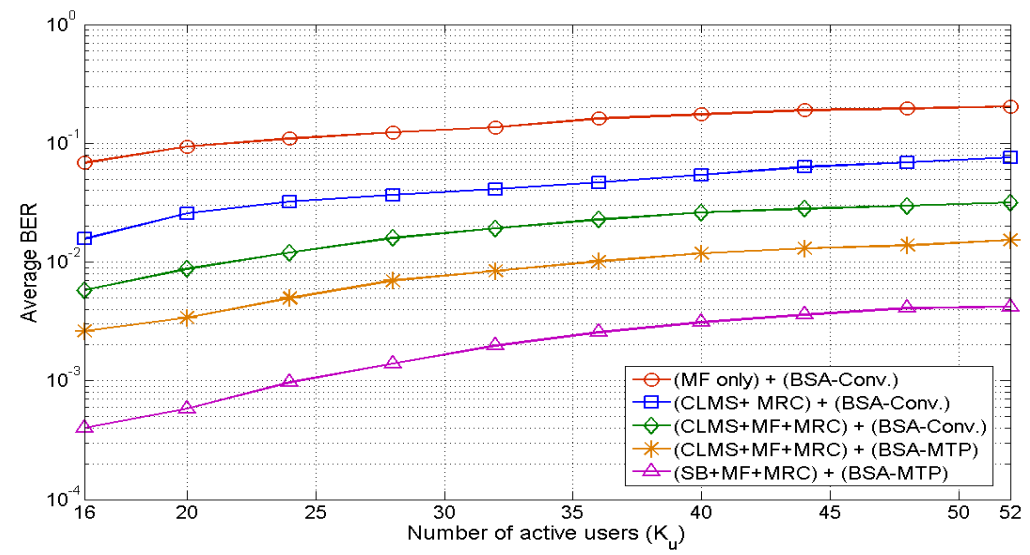

Figure 9. Average BER versus $K_{u}$ for $\sigma_{v}^{2}=4 \mathrm{~dB}$ and $\mathrm{SNR}=10 \mathrm{~dB}$

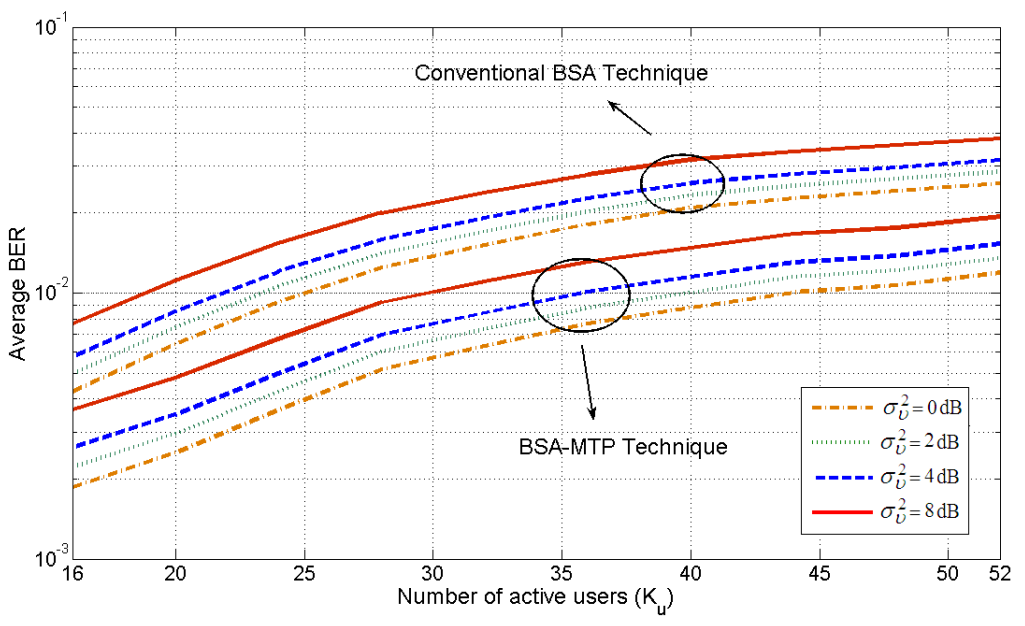

Figure 10. Average BER of all users versus $K_{u}$ for different values of $\sigma_{v}^{2}$

technique support $K_{u}=22$ users. In addition, the figure shows that the average BER in the CLMS algorithm is higher than the SB technique. For example, At a BER of 0.003 and for the BSA-MTP technique, SB, MF, and MRC receiver support $K_{u}=40$ users, while CLMS, MF, and MRC receiver support $K_{u}=18$ users, respectively. Also it can be seen that the three-stage RAKE receiver can achieve lower BER than the other receivers. It should be mentioned that 
International Journal of Computer Networks \& Communications (IJCNC) Vol.3, No.1, January 2011

increasing the number of active users $\left(K_{u}\right)$, will increase the number of users that can be transferred to other base stations $\left(K_{r}\right)$ in the BSA-MTP technique.

Figure 10 presents the average BER versus the number of active users as Figure 8, for SNR $=10 \mathrm{~dB}$ and different values of $\sigma_{v}^{2}$. Similar to Figure 8 , we observe that the average BER for $\sigma_{v}^{2}=0 \mathrm{~dB}$ is lower than $\sigma_{v}^{2}=2,4,8 \mathrm{~dB}$. For example, at a BER of 0.01 and for the BSAMTP technique, the three-stage RAKE receiver with $\sigma_{v}^{2}=0 \mathrm{~dB}$ support $K_{u}=44$ users, while for $\sigma_{v}^{2}=2,4,8 \mathrm{~dB}$ support $K_{u}=40,36$, and 30 users, respectively. Accordingly in this case, with $\sigma_{v}^{2}$ from 2 to $8 \mathrm{~dB}$, the system capacity degrades from $9 \%$ to $32 \%$ compared to the case of perfect power control.

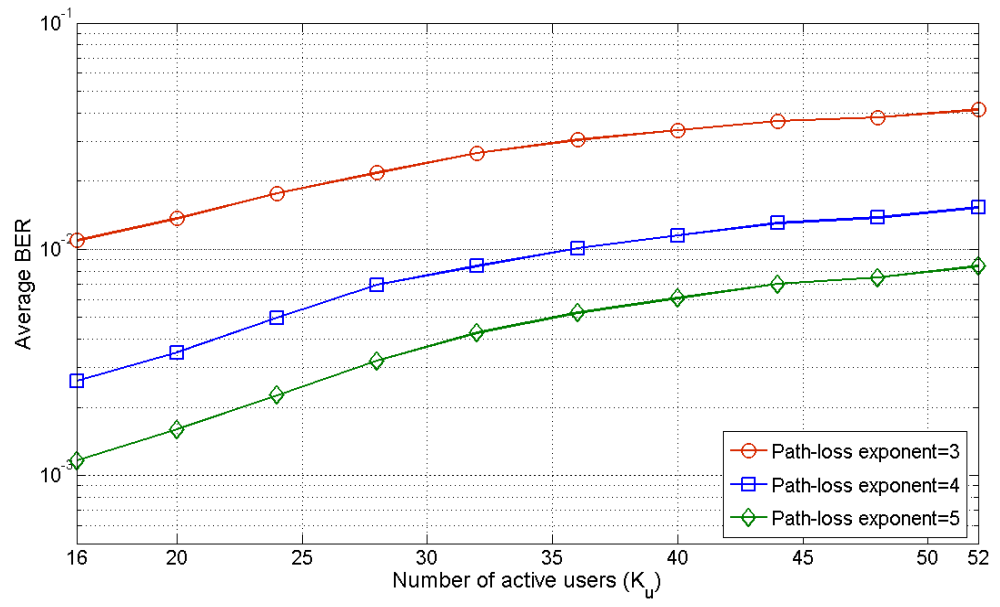

Figure 11. Influence of path-loss exponent on average BER $\left(\sigma_{v}^{2}=4 \mathrm{~dB}, \mathrm{SNR}=10 \mathrm{~dB}\right)$

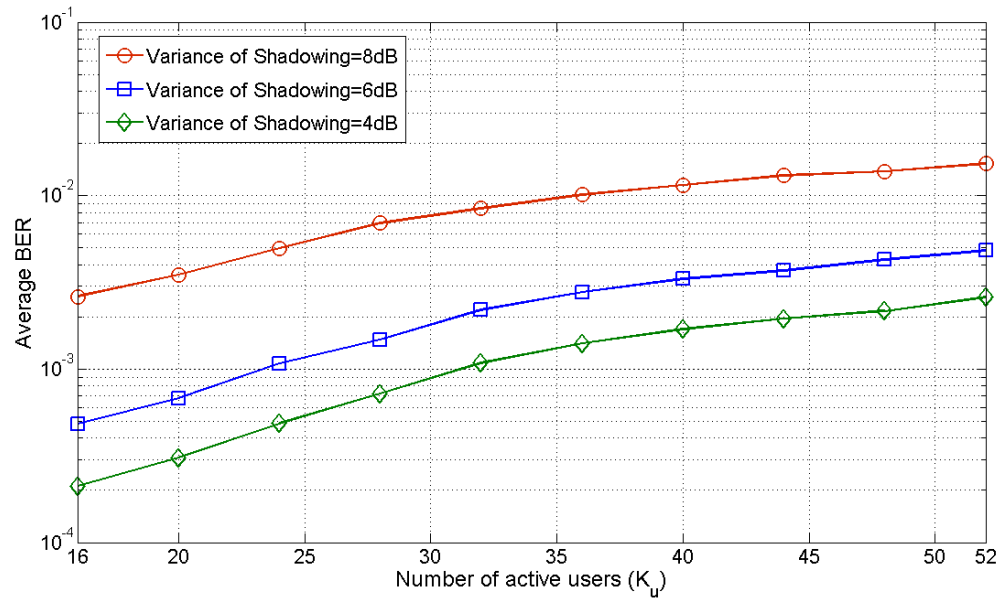

Figure 12. Influence of variance of shadowing on average BER $\left(\sigma_{v}^{2}=4 \mathrm{~dB}, \mathrm{SNR}=10 \mathrm{~dB}\right)$

Other results displayed in Figures 11 and 12 show the influence of channel propagation conditions (path-loss exponent, $L_{\alpha}$, and variance of shadowing, $\sigma_{\xi}^{2}$ ) on the average BER for the CLMS, MF, and MRC receiver and the BSA-MTP technique. In this simulations, we assume $\mathrm{SNR}=10 \mathrm{~dB}$ and $\sigma_{v}^{2}=4 \mathrm{~dB}$. In Figure 11, we can observe that, as expected, an increase in the 
path-loss exponent entails a decrease in the MAI level and, therefore, an improvement in system performance. For example, at a BER of 0.01, capacity is, respectively, 16, 36 users for $L_{\alpha}=3$ and 5. In Figure 12, it is seen that if $\sigma_{\xi}^{2}$ increases from 6 to $8 \mathrm{~dB}$, the number of active users decreases by approximately $55 \%$ for a required average BER of 0.0025 , whereas if $\sigma_{\xi}^{2}$ increases from 4 to $6 \mathrm{~dB}$, the capacity decreases by approximately $32 \%$.

Finally, Figure 13 shows curves of the average BER versus the number of active users for $\sigma_{v}^{2}=4 \mathrm{~dB}, \mathrm{SNR}=10 \mathrm{~dB}$, and different values of the number of propagation paths $(L)$. It can be seen that for $B E R=0.003$, the number of users allowed in the system increases from 18 to 35 when $L$ varies from 2 to 4 .

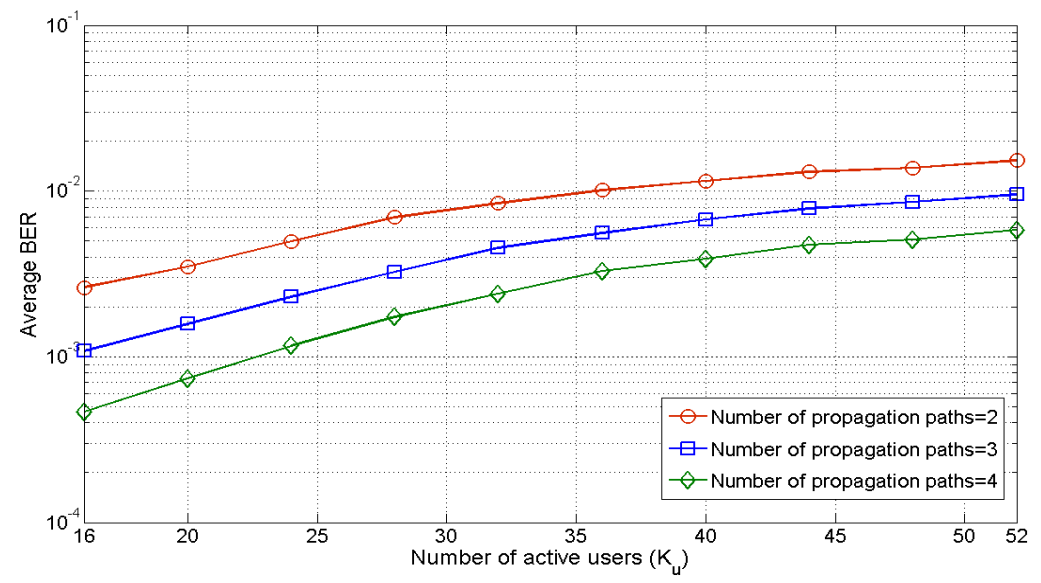

Figure 13. Average BER for different values of the number of propagation paths $\left(\sigma_{v}^{2}=4 \mathrm{~dB}\right.$, $\mathrm{SNR}=10 \mathrm{~dB}$ )

\section{CONClusions}

In this paper, we studied the RAKE receiver performance of multiple-cell DS-CDMA system with the space diversity processing, Rayleigh frequency-selective channel model, power control error, and base station assignment. This receiver consists of three stages. In the first stage, with the CLMS algorithm, the desired users' signal in an arbitrary path is passed and the IPI is reduced in other paths in each RAKE finger. Also in this stage, the MAI from other users is reduced. Thus, the MF can be used for the MAI reduction in each RAKE finger in the second stage. Also in the third stage, the output signals from the matched filters are combined according to the conventional MRC principle and then are fed into the decision circuit for the desired user.

Accordingly, we proposed BSA-MTP technique to reduce the CCI and the MAI in DS-CDMA cellular systems. It has been shown that, by using antenna arrays at the base stations, the proposed technique will decrease the average BER of the system to support a significantly larger number of users. It has also been observed that the average BER in three-stage RAKE receiver is less than the one- and two-stage RAKE receivers. On the other hand, it has been shown that the average BER in the SB technique is lower than the CLMS algorithm. Also our simulations show that the variations in power level due to PCE have a detrimental effect on system performance. 
International Journal of Computer Networks \& Communications (IJCNC) Vol.3, No.1, January 2011

\section{ACKNOWLEDGEMENT}

This research was supported by the Islamic Azad University, Qazvin Branch, Qazvin, Iran.

\section{REFERENCES}

[1] R. L. Peterson, R. E. Ziemer, and D. E. Borth, Spread-Spectrum Communications. Prentice-Hall, 1995.

[2] W. Ye and A. M. Haimovich, "Performance of cellular CDMA with cell site antenna array, Rayleigh fading, and power control error," IEEE Transactions on Communications, vol. 48, no. 7, pp. 1151-1159, July 2000.

[3] A. Abrardo and D. Sennati, "On the analytical evaluation of closed-loop power-control error statistics in DS-CDMA cellular systems," IEEE Transactions on Vehicular Technology, vol. 49, no. 6, pp. 2071-2080, Nov. 2000.

[4] L. Carrasco and G. Femenias, "Reverse link performance of a DS-CDMA system with both fast and slow power controlled users," IEEE Transactions on Wireless Communications, vol. 7, no. 4, pp. 1255-1263, Apr. 2008.

[5] L. Qian and Z. Gajic, "Variance minimization stochastic power control in CDMA system," IEEE Transactions on Wireless Communications, vol. 5, no. 1, pp. 193-202, Jan. 2006.

[6] M. Rintamaki, H. Koivo, and I. Hartimo, "Adaptive closed-loop power control algorithms for CDMA cellular communication systems," IEEE Transactions on Vehicular Technology, vol. 53, no. 6, pp. 1756-1768, Nov. 2004.

[7] J. Wang and A. Yu, "Open-loop power control error in cellular CDMA overlay systems," IEEE Journal on Selected Areas in Communications, vol. 19, no. 7, pp. 1246-1254, July 2001.

[8] J. T. Wang, "Admission control with distributed joint diversity and power control for wireless networks,” IEEE Transactions on Vehicular Technology, vol. 58, no. 1, pp. 409-419, Jan. 2009.

[9] K. Kumatani, J. McDonough, B. Rauch, D. Klakow, P. N. Garner, and W. Li, "Beamforming with a maximum negentropy criterion," IEEE Transactions on Audio, Speech, and Language Processing, vol. 17, no. 5, pp. 994-1008, July 2009.

[10] J. Chang, L. Tassiulas, and F. Rashid-Farrokhi, "Joint transmitter receiver diversity for efficient space division multiaccess," IEEE Transactions on Wireless Communications, vol. 1, no. 1, pp. 16-27, Jan. 2002.

[11] F. Rashid-Farrokhi, L. Tassiulas, and K. J. Ray-Liu, "Joint optimal power control and beamforming in wireless networks using antenna arrays," IEEE Transactions on Communications, vol. 46, no. 10, pp. 1313-1324, Oct. 1998.

[12] R. D. Yates and C. Huang, "Integrated power control and base station assignment," IEEE Transactions on Vehicular Technology, vol. 44, no. 3, pp. 638-644, Aug. 1995.

[13] S. V. Hanly, "An algorithm for combined cell-site selection and power control to maximize cellular spread spectrum capacity," IEEE Journal on Selected Areas in Communications, vol. 13, no. 7, pp. 1332-1340, Sep. 1995.

[14] M. Mahmoudi and E. S. Sousa, "Joint power control, base station assignment and sectorization for CDMA cellular systems," in Proc. 2000 IEEE Vehicular Technology Conference, Boston, MA, vol. 2, pp. 573-580, Sep. 24-28, 2000.

[15] M. Dosaranian-Moghadam, H. Bakhshi, and G. Dadashzadeh, "Interference management for DS-CDMA receiver through base station assignment in multipath fading channels," in Proc. 2010 IEEE International Conference on Wireless Communications, Networking and Information Security, Beijing, China, pp. 257-263, June 2010.

[16] M. Dosaranian-Moghadam, H. Bakhshi, and G. Dadashzadeh, "Joint constrained LMS algorithm and base station assignment for DS-CDMA receiver in multipath fading channels," Accepted for 
International Journal of Computer Networks \& Communications (IJCNC) Vol.3, No.1, January 2011

publication in the IEEE Wireless Communications, Networking and Mobile Computing, Chengdu, China, Sep. 23-25, 2010.

[17] M. Dosaranian-Moghadam, H. Bakhshi, and G. Dadashzadeh, "Interference management for DS-CDMA systems through closed-loop power control, base station assignment, and beamforming," Journal of Wireless Sensor Network, vol. 2, no. 6, pp. 472-482, June 2010.

[18] N. A. Mohamed and J. G. Dunham, "A low-complexity combined antenna array and interference cancellation DS-CDMA receiver in multipath fading channels," IEEE Journal on Selected Areas in Communications, vol. 20, no. 2, pp. 248-256, Feb. 2002.

[19] N. A. Mohamed and J. G. Dunham, "Adaptive beamforming for DS-CDMA using conjugate gradient algorithm in a multipath fading channel," in Proc. 1999 IEEE Emerging Technologies Symp., Dallas, TX, pp. 859-863, Apr. 12-13, 1999.

[20] F. Rashid-Farrokhi, K. J. Ray-Liu, and L. Tassiulas, "Transmit beamforming and power control for cellular systems," IEEE Journal on Selected Areas in Communications, vol. 16, no. 8, pp. 1437-1450, Oct. 1998.

[21] J. Litva and T. Kwok-Yeung, Digital Beamforming in Wireless Communications. Artech-House, 1996.

[22] S. Xinyu, L. Xiaohua, and Z. Jianjiang, "Robust adaptive beamforming based on maximum likelihood estimation," International Conference on Microwave and Millimeter Wave Technology, vol. 3, pp. 1137-1140, Apr. 2008.

[23] M. Z. Shakir and T. S. Durrani, "Narrowband beamforming algorithm for smart antennas," International Bhurban Conference on Applied Sciences \& Technology, pp. 49-54, Jan. 2007.

[24] S. Haykin, Adaptive filter theory. $3^{\text {th }}$ ed., New Jersey: Prentice Hall, 1996.

[25] N. Kong and L. B. Milstein, "Average SNR of a generalized diversity selection combining scheme," IEEE Communications Letters, vol. 3, no. 3, pp. 57-59, Mar. 1999.

[26] J. M. Romero-Jerez, C. Tellez-Labao, and A. Diaz-Estrella, "Effect of power control imperfections on the reverse link of cellular CDMA networks under multipath fading," IEEE Transactions on Vehicular Technology, vol. 53, no.1, pp. 61-71, Jan. 2004.

[27] J. C. Liberti and T. S. Rappaport, Smart Antennas for Wireless Communications IS -95 and Third Generation CDMA Applications. Prentice-Hall, 1999.

[28] M. Dosaranian-Moghadam, H. Bakhshi, G. Dadashzadeh, and M. Godarzvand-Chegini, "Joint base station assignment, power control error, and adaptive beamforming for DS-CDMA cellular systems in multipath fading channels," Accepted for publication in IEEE Global Mobile Congress, Shanghai, China, Oct. 18-19, 2010.

[29] B. Allen and M. Beach, "On the analysis of switched-beam antennas for the W-CDMA downlink”, IEEE Transactions on Vehicular Technology, vol. 53, no. 3, pp. 569-578, May 2004.

[30] M. Dosaranian-Moghadam, H. Bakhshi, and G. Dadashzadeh, "Joint centralized power control and cell sectoring for interference management in CDMA cellular systems in a 2D urban environment," Journal of Wireless Sensor Network, vol. 2, no. 8, pp. 599-605, Aug. 2010.

\section{Authors}

Mohamad Dosaranian Moghadam was born in Tehran, Iran on May 26, 1979. He received the B.Sc. degree in electrical engineering from Islamic Azad University, Qazvin Branch, Qazvin, Iran and the M.Sc. degree in communication engineering from Ferdowsi University, Mashad, Iran, in 2002 and 2005, respectively. He is currently working toward the Ph.D degree in the Department of Electrical Engineering, Islamic Azad University, Science \& Research Branch, Tehran, Iran.

His research interests include power control, wireless communications, array

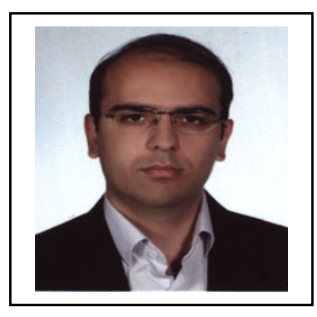
and statistical signal processing, smart antennas, and adaptive filtering. 
Hamidreza Bakhshi was born in Tehran, Iran on April 25, 1971. He received the B.Sc. degree in electrical engineering from Tehran University, Iran in 1992, the M.Sc. and Ph.D. degree in Electrical Engineering from Tarbiat Modarres University, Iran in 1995 and 2001, respectively. Since 2001, he has been as an Assistant Professor of Electrical Engineering at Shahed University, Tehran, Iran.

His research interests include wireless communications, multiuser detection, and smart antennas.

Gholamreza Dadashzadeh was born in Urmia, Iran, in 1964. He received the B.Sc. degree in communication engineering from Shiraz University, Shiraz, Iran in 1992 and M.Sc. and Ph.D. degree in communication engineering from Tarbiat Modarres University (TMU), Tehran, Iran, in 1996 and 2002, respectively. From 1998 to 2003, he has worked as head researcher of Smart Antenna for Mobile Communication Systems (SAMCS) and WLAN 802.11 project in radio communications group of Iran Telecomm Research Center (ITRC). From 2004 to 2008, he was dean of Communications Technology Institute (CTI) in ITRC. He is currently as

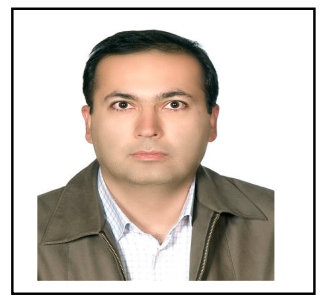
Assistance Professor in the Department of Electrical Engineering at Shahed University, Tehran, Iran. He is a member of IEEE, Institute of Electronics, Information and Communication Engineers (IEICE) of Japan and Iranian Association of Electrical and Electronics Engineers (IAEEE) of Iran. $\mathrm{He}$ honored received the first degree of national researcher in 2007 from Iran's ministry of ICT.

His research interests include antenna design and smart antennas. 